\title{
OPERATIONAL PROPERTIES OF ANTI-GRAFFITI COATING SYSTEMS FOR ROLLING STOCK
}

\author{
Łukasz Pasieczyński ${ }^{1}$, Norbert Radek², Jolanta Radziszewska-Wolińska ${ }^{3}$ \\ 1 Firma Handlowa Barwa Jarosław Czajkowski, ul. Warkocz 3-5, 25-253 Kielce, Poland, e-mail: Ipasieczynski@ \\ barwa.kielce.pl \\ 2 Kielce University of Technology, Faculty of Mechatronics and Machine Building, Al. 1000-lecia P. P. 7, 25-314 \\ Kielce, Poland, e-mail: norrad@tu.kielce.pl \\ ${ }^{3}$ The Railway Institute, Head of Materials \& Structure Laboratory, ul. Chłopickiego 50, 04-275 Warszawa, \\ Poland, e-mail: JRadziszewska-Wolinska@ikolej.pl
}

Received: 2017.11 .06

Accepted: 2018.02.01

Published: 2018.03.01

\begin{abstract}
The article presents the comparative tests results of selected properties of anti-graffiti paint system for rolling stock industry. The measurements included an adhesion test, free surface energy, surface geometric structure, corrosion resistance and flame resistance test. The system consists of high solid corrosion protection primer, putty, filler, basecoat and anti-graffiti clearcoat.
\end{abstract}

Keywords: corrosion protection, antigraffiti, coating properties, rolling stock

\section{INTRODUCTION}

Paint systems for rolling stock must fulfill mechanical and qualitative properties to protective and decorative properties maintenance longer on the vehicle. These requirements include: adhesion, resistance to weather conditions (humidity, UV, corrosion) as well as hardness and specialized properties such as anti-graffiti. Coating systems intended for rolling stock in addition to the above-mentioned requirements, as well as ease of application and operation, must also have adequate fire performance [12].

The study of wettability and free surface energy is of interest to many disciplines [14]: physics, chemistry, materials engineering and biotechnology. Wettability of materials used in the industry by various liquids is of great practical importance in industrial processes such as bonding, sealing, painting, printing and application of various protective coatings. The paper examines the anti-adhesion properties of subsequent undesirable coatings onto the antigraffiti coating, determining the wettability of these varnishes.
At the next stage of the study, measurements of the surface geometric structure of the anti-graffiti coating system were performed. The surface geometric structure (SGS) substantially influences many processes that occur in the outer layer. A lot of publications deal with the measurement methods and the assessment of surface roughness and waviness $[1,2,7,11,15]$. Analysis of properties of anti-graffiti coating systems requires many methods $[8,9]$.

Another important issue is to protect paintings before end users and to ensure proper operation of the vehicle. Therefore, owner of the rolling stock should follow the manufacturer's instructions, how to handle with them and what may prolong the life of the product for next years. Application of various types of paints (graffiti) on the vehicle may lead to shortening durability of the coating surface. Spray paints should be treated as dangerous substances for coatings because they contain various solvents and other substances that might soften or migrate into protective coating and cause delamination of the coating system. Graffiti paints that are difficult to remove require the use of more aggres- 
sive materials, which increase the possibility of mechanical or chemical damage of the coating system and consequently reduce the thickness of the protective coating or remove it completely. In addition, aggressive chemicals removers are dangerous to the users and environment. The article presents the comparative tests results of selected properties of anti-graffiti paint system for rolling stock industry.

\section{MATERIALS AND TREATMENT PARAMETERS}

Coatings was applied with a SATA spray gun on S235 carbon steel, before the application the surface of steel was polished with 80-grit sandpaper. Coating system consisting of the following layers: anti-corrosion epoxy primer, repair filler, primer filler, basecoat and anti-graffiti clearcoats XPC60011, XPC60012, XPC60036, BO100AGR. Each layer is applied and dried in accordance with the requirements of the technological cards. The prepared samples were conditioned at $23{ }^{\circ} \mathrm{C}$ and $50 \%$ humidity for minimum 7 days in order to perform tests on dry coating.

\section{RESULTS AND DISCUSSION}

\section{Operational tests}

Cured coating was also tested for mechanical properties and corrosion resistance. After conditioning period had finished, thickness of dry coating was tested by magnetic induction method, adhesion tests were performed by pulloff test. Hardness of cured coating was measured by Koenig pendulum. Summary tests results are presented in Table 1.

Also measurements of "orange peel" was made, which is responsible for the final appearance of the coating. When measuring device's laser beam optically scans the coating surface undulation, like the eyes captures the dark-light pat- tern reflections. Results are shown in five wavelength ranges from 0.1 to $30 \mathrm{~mm}$ Table 2 .

The measurements of gloss and color parameters was conducted using Byk micro-Tri-gloss and Minolta Spectrophotometer CM-600d on the samples after removing graffiti from coating surface. The measurements were performed both before application of graffiti after cleaning with water and additionally after cleaning using solvent nitro. The results are summarized in Table 3 . After the test, the sample was dried, and the measurements of gloss and color parameters $-L^{*}, a^{*}$, $b^{*}$ for light D65. The results are shown together with diagrams of color change. The color change is expressed as $\Delta E$, which is defined by the equation (1):

$$
\Delta E=\sqrt{\left(\Delta L^{*}\right)^{2}-\left(\Delta a^{*}\right)^{2}+\left(\Delta b^{*}\right)^{2}}
$$

where: $\Delta E-$ color difference expressed as the distance between two points in three-dimensional space,

$\Delta L^{*}$ - the difference in distance between two points in the dimension $\mathrm{L}$ - brightness, $\Delta a^{*}$ - the difference in distance between two points in dimension a - green and red color,

$\Delta b^{*}$ - the difference in distance between two points in the dimension $\mathrm{b}-$ blue and yellow color.

The next test of corrosion resistance to aggressive environmental conditions were finished and assessed after 1000 hours of exposure. The corrosion resistance test was performed in the salt spray chamber according to PN-EN ISO 9227 at $35{ }^{\circ} \mathrm{C}$ using $5 \%$ saline solution. The result revealed no changes in the tested coatings (no blistering, cracking, corrosion or thread-like corrosion). Given time of 1000 hours is a minimum for the coating to be resistant to environmental effects (Table 4). Figure 1 shows an example a view of sample after corrosion resistance test for anti-graffiti BO100-AGR. However, this time could be extended when aluminum is used as a substrate instead of steel.

Table 1. Selected properties of dry coating applied on steel

\begin{tabular}{|l|c|c|c|}
\hline \multicolumn{1}{|c|}{ System } & $\begin{array}{c}\text { Adhesion test [MPa] } \\
\text { PN-EN ISO 4624 }\end{array}$ & $\begin{array}{c}\text { Pendulum Hardness [s] } \\
\text { PN-EN ISO 1522 }\end{array}$ & $\begin{array}{c}\text { Thickness [ } \mu \mathrm{m}] \\
\text { PN-EN ISO 2808 }\end{array}$ \\
\hline XPC60011 & 2.28 & 95 & 2210 \\
\hline XPC60012 & 2.18 & 84 & 2190 \\
\hline XPC60036 & 2.24 & 110 & 2270 \\
\hline BO100-AGR & 2.22 & 121 & 2240 \\
\hline
\end{tabular}


Table 2. Orange peel measurement results

\begin{tabular}{|c|c|c|c|c|c|}
\hline \multirow{2}{*}{$\begin{array}{c}\text { The parameter } \\
\text { (corresponding to length } \\
\text { of surface undulation) }\end{array}$} & \multirow{2}{*}{ Range [mm] } & \multicolumn{4}{|c|}{ Results } \\
\cline { 3 - 5 } & & XPC60011 & XPC60012 & XPC60036 & BO100-AGR \\
\hline $\mathrm{du}$ & $0.1<$ & 10.2 & 13.1 & 11.5 & 8.3 \\
\hline $\mathrm{Wa}$ & $0.1-0.3$ & 27.0 & 32.0 & 32.9 & 27.7 \\
\hline $\mathrm{Wb}$ & $0.3-1$ & 45.9 & 56.0 & 52.9 & 44.7 \\
\hline $\mathrm{Wc}$ & $1-3$ & 42.7 & 58.5 & 44.4 & 25.0 \\
\hline Wd & $3-10$ & 49.6 & 44.4 & 40.2 & 24.9 \\
\hline We & $10-30$ & 27.4 & 30.7 & 26.5 & 18.7 \\
\hline LW & $1.2-12$ & 70.3 & 97.3 & 63.6 & 38.4 \\
\hline SW & $0.3-1.2$ & 41.5 & 48.0 & 34.7 & 13.8 \\
\hline DOI & - & 84.6 & 80.6 & 82.4 & 85.8 \\
\hline
\end{tabular}

Table 3. Change of color and gloss parameter after cleaning for BO100-AGR

\begin{tabular}{|c|c|c|c|}
\hline Sample & Medium & Gloss change & Color change \\
\hline 1 & Water & No loss of gloss & $\begin{array}{l}\text { 1. } \Delta \mathrm{E}=0.44 \\
\text { 2. } \Delta \mathrm{E}=0.32 \\
\text { 3. } \Delta \mathrm{E}=0.61\end{array}$ \\
\hline 2 & Nitro solvent & No loss of gloss & $\begin{array}{l}\text { 1. } \Delta \mathrm{E}=0.67 \\
\text { 2. } \Delta \mathrm{E}=0.38 \\
\text { 3. } \Delta \mathrm{E}=0.43\end{array}$ \\
\hline
\end{tabular}

Table 4. Results after corrosion resistance test for BO100-AGR

\begin{tabular}{|l|c|}
\hline Exposure to salt spray $[\mathrm{h}]$ & 1000 \\
\hline Resistance to salt spray $[\mathrm{h}]$ & $>1000$ \\
\hline Degree of blistering & 0 \\
\hline Degree of corrosion & 0 \\
\hline Degree of exfoliation & 0 \\
\hline Degree of cracking & 0 \\
\hline Degree of delamination around of crack [mm] & 2 \\
\hline Degree of corrosion around of crack [mm] & 0.3 \\
\hline
\end{tabular}

\section{Measurement of contact angle and free surface energy}

One of the most commonly used methods of determining the contact angle of a material [14] is a method based on the geometry of the droplet (Figure 2). The surface of the droplet is most often in the shape of a circular arc, and then the contact angle is calculated from the measurement of the height $h$ and the radius of the contact surface of the drop $r$. The height of the circular arc is given by $\mathrm{h}=\mathrm{R}(1-\cos \Theta)$ and the surface contact radius $r=R \sin \Theta$. From these relationships we get a equation (2):

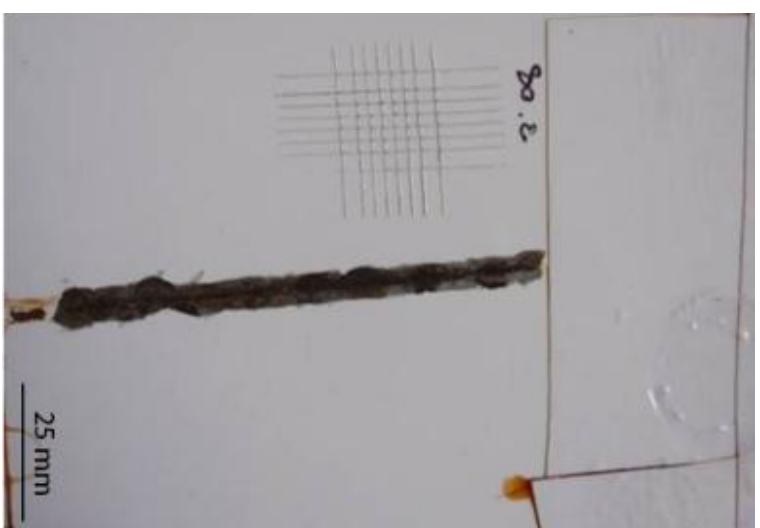

Fig. 1. View of sample after corrosion resistance test

$$
\Theta=\frac{2 h}{r}
$$

where: $h$ - height of circular arc,

$r$ - the radius of the contact surface of the drop.

The value of free surface energy of construction materials is determined indirectly by measuring the contact angles of selected measuring fluids. Distilled water and diododomethane (DIM) are used to measure the contact angle. The stereoscopic microscope with the camera and the MicroScan v 1.3 software were used for droplet observation and contact angle measurement. 
a)

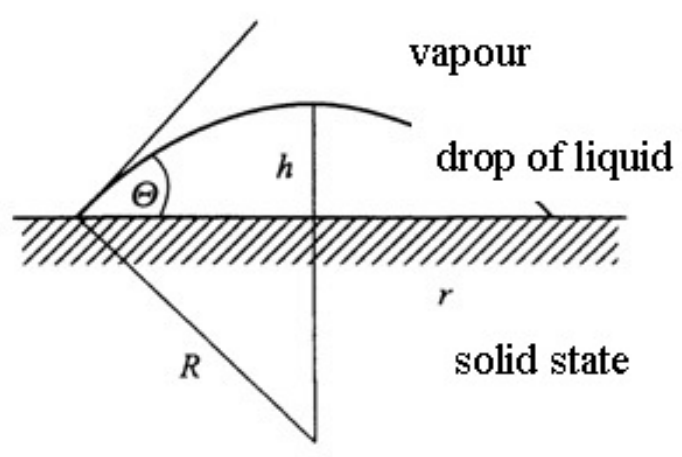

b)

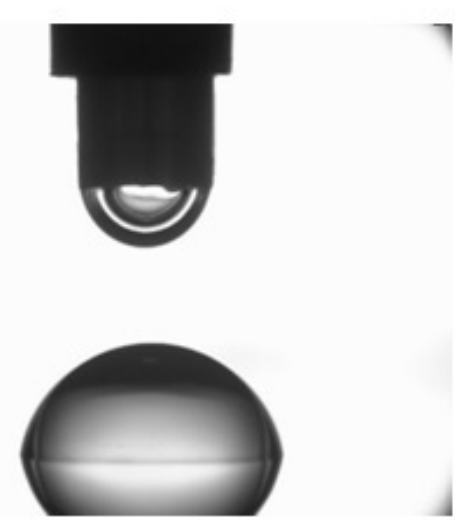

Fig. 2. Measurement of the contact angle of the drop geometry (a), view of the drop of a measuring liquid (b)

The following values of free surface energy constants of the measuring fluids and their polar and dispersion components were assumed: $\gamma_{\mathrm{w}}=72.8 \quad\left[\mathrm{~mJ} / \mathrm{m}^{2}\right], \gamma_{\mathrm{w}}^{\mathrm{p}}=51.0 \quad\left[\mathrm{~mJ} / \mathrm{m}^{2}\right], \gamma_{\mathrm{w}}^{\mathrm{d}}=21.8$ $\left[\mathrm{mJ} / \mathrm{m}^{2}\right], \quad \gamma_{\mathrm{d}}=50.8 \quad\left[\mathrm{~mJ} / \mathrm{m}^{2}\right], \quad \gamma_{\mathrm{d}}^{\mathrm{p}}=2.3 \quad\left[\mathrm{~mJ} / \mathrm{m}^{2}\right]$, $\gamma_{\mathrm{d}}^{\mathrm{d}}=48.5\left[\mathrm{~mJ} / \mathrm{m}^{2}\right]$. The measuring liquid was applied to the test surface with a $5 \mu$ constant volume micropipette.

One of the most commonly used methods for determining free surface energy is the OwensWendt method $[6,14,16]$ in which it is assumed that the free surface energy is the sum of two components, dispersion and polar:

$$
\gamma_{s}=\gamma_{s}^{d}+\gamma_{s}^{p}
$$

where: $\gamma_{s}^{d}-d$ ispersion component of free surface energy,

$\gamma_{S}^{p}-$ polar component of free surface energy.

The minimum number of contact angle measurement on each sample with another anti-graffiti coating system was 10 times, which allowed for averaging the test results.

Free surface energy (FSE) values were determined by measuring the contact angle. FSE estimation was done at least six times for each surface. Table 5 summarizes the averaged wetting angle and free surface energy measurements of the anti-graffiti coating systems.

The obtained results show that the lowest value of free surface energy was obtained by anti-graffiti BO100-AGR coating system. Also the highest values of measured wetting angles with distilled water and diododomethane were the BO100-AGR system. Analyzing the obtained results, we can observe high repeatability of the measurements, as evidenced by small values of standard deviations (Table 5).

\section{Measurement of flammability properties}

The fire properties for coatings intended for the vehicle's body of rolling stock in accordance with the European requirements of PN EN 45545-2 [10], valid from 2016, are characterized by the following parameters:

- CFE (critical heat flux) $\left[\mathrm{kW} / \mathrm{m}^{2}\right]$ according ISO 5658-2 [4],

- MARHE (maximum average rate of heat release) $\left[\mathrm{kW} / \mathrm{m}^{2}\right]$ according ISO 5660-1 [5],

- $\mathrm{Ds}_{(\max )}$ (maximum optical density) [-]according EN ISO 5659-2 [3],

- $\mathrm{CIT}_{\mathrm{G}}$ (standard toxicity index) [-]according EN ISO 5659-2 [3] and PN EN 45545-2 [10] Annex C.

Table 5. Results of contact angle measurements and free surface energy of anti-graffiti coating systems

\begin{tabular}{|c|c|c|c|c|c|c|}
\hline \multirow{3}{*}{ System } & \multicolumn{4}{|c|}{ Contact angle [ ${ }^{\circ}$ ] } & \multirow{3}{*}{$\begin{array}{c}\text { Free surface } \\
\text { energy } \\
\gamma_{S}\left[\mathrm{~mJ} / \mathrm{m}^{2}\right]\end{array}$} & \multirow{3}{*}{$\begin{array}{c}\text { Standard } \\
\text { deviation } \\
\gamma_{S}\left[\mathrm{~mJ} / \mathrm{m}^{2}\right]\end{array}$} \\
\hline & \multicolumn{2}{|c|}{ average } & \multicolumn{2}{|c|}{ Standard deviation } & & \\
\hline & $\begin{array}{c}\text { Distilled } \\
\text { water }\end{array}$ & Diododomethane & $\begin{array}{c}\text { Distilled } \\
\text { water }\end{array}$ & Diododomethane & & \\
\hline XPC 60011 & 78.55 & 30.52 & 0.45 & 1.49 & 49.87 & 3.14 \\
\hline XPC 60012 & 80.63 & 28.80 & 1.95 & 2.17 & 52.30 & 0.87 \\
\hline XPC 60036 & 75.58 & 24.23 & 0.73 & 1.20 & 55.85 & 0.55 \\
\hline BO100-AGR & 80.63 & 40.48 & 1.71 & 0.66 & 48.15 & 0.69 \\
\hline
\end{tabular}


The previous tests of the paint systems showed that the first two parameters were the most difficult to fulfill, so the tests were started from this two parameters. The tests were performed at the Research Institute for Materials and Design Elements of the Railway Institute in Warsaw.

Side flame spread test was performed by exposure a vertically arranged sample to external heat flux with a standardized density distribution $\left(10.9\right.$ to $\left.1.1 \mathrm{~kW} / \mathrm{m}^{2}\right)$. The flame spreads along the sample in a direction consistent with the diminishing heat flux until the spontaneous extinguishment or completion of the test duration. At time of measurement, the flame transit time in the central part of the sample will be recorded through the zones and the $\mathrm{Qsb}\left[\mathrm{MJ} / \mathrm{m}^{2}\right]$ flame retaining heat. At the end of the test, the length of the burned part of the sample is determined and entered into the program to calculate the critical heat flux CFE $\left[\mathrm{kW} / \mathrm{m}^{2}\right]$ by the control program.

Measurements of maximum heat generated were made using a conical calorimeter using the oxygen consumption calorimetry principle: For most materials per kilogram of oxygen consumed by burning material, 13.1 MJ of heat is released to within $\pm 5 \%$. The use of precision oxygen measurement equipment in gas extraction and gas flow measurement in the extract allows for a very precise determination of oxygen consumption and at the same time to accurately calculate the value of the released heat and its rate of release.
Samples with an anti-graffiti coating system were treated with a cone-shaped electric radiator with a radiation intensity of $50 \mathrm{~kW} / \mathrm{m}^{2}$. The ignition was initiated by the spark and combustion was carried out in air atmosphere $(0.024$ $\mathrm{m}^{3} / \mathrm{s}$ ). Classification according to EN 45545-2 [10] defines the MARHE parameter, which represents the maximum average rate of heat release (ARHE) over a 20 minute test. The ARHE value is calculated according to the following formula:

$$
\operatorname{ARHE}\left(t_{n}\right)=\frac{\sum_{2}^{n}\left(t_{n}-t_{n-1}\right) \times \frac{q_{n+} q_{n-1}}{2}}{t_{n}-t_{n-1}}
$$

where: $t$ - time, mostly $t_{1}=0$

$$
q \text { - the rate of heat release, mostly } q_{1}=0
$$

Figure 3 shows an example HRRs heat releasing curve for anti-graffiti BO100-AGR. Table 6 summarizes the results of the flammability tests of anti-graffiti coating systems.

From the analysis of Table 6, it is clear that the final values slightly differ from each other. The graphs and observations of the combustion processes also show their similarity and lack of significant impact on the course of the anti-graffiti layer used. This is due to the fact that in the tests carried out, the samples were subjected to radiation with a radiation intensity of $50 \mathrm{~kW} / \mathrm{m}^{2}$, simulating the conditions of the fire $[12,13]$. As a result, all layers of the coating system burned down.

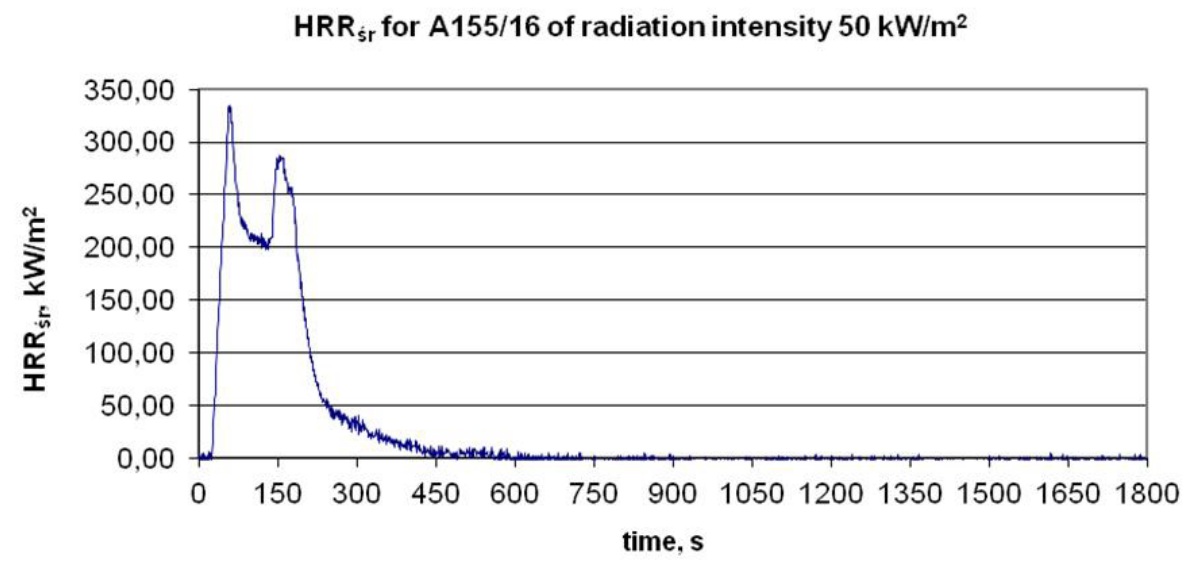

Fig. 3. HRRs heat releasing curve for anti-graffiti BO100-AGR

Table 6. Summary of fire test results

\begin{tabular}{|c|c|c|c|c|}
\hline \multirow{2}{*}{ Parameter } & \multicolumn{4}{|c|}{ System } \\
\cline { 2 - 5 } & XPC 60011 & XPC 60012 & XPC 60036 & BO100-AGR \\
\hline CFE $\left[\mathrm{kW} / \mathrm{m}^{2}\right]$ & 11.5 & 11.7 & 11.1 & 12.4 \\
\hline MARHE $\left[\mathrm{kW} / \mathrm{m}^{2}\right]$ & 177.6 & 167.9 & 178.4 & 198.1 \\
\hline
\end{tabular}




\section{Measurements of the surface geometric structure}

Measurements of surface geometric structure were carried out at the Laboratory of Computer Measurements of Geometric Quantities of the Kielce University of Technology. Tests were performed using a Talysurf CCI optical profilometer using the coherent correlation interferometry method, enabling a resolution of $0.01 \mathrm{~nm}$ with a $\mathrm{z}$ axis resolution. The measurement result is recorded in a matrix of $1024 \times 1024$ measuring points using the $\mathrm{x} 10$ lens, giving a measured area of $1.65 \mathrm{~mm} \times 1.65 \mathrm{~mm}$ and a horizontal resolution of $1.65 \mu \mathrm{m} \times 1.65 \mu \mathrm{m}$. Ten measurements were made on samples of anti-graffiti and S355 steel, allowing averaging of the results. The obtained images of surface stereometry and their analysis using the software TalyMap Platinium allowed to evaluate the geometrical structure of the examined surfaces.

Figure 4 shows a sample isometric roughness of the surface of the anti-graffiti XPC 60012 coating system, while Figure 5 shows the isometric image of the wavy surface of the coating system. Table 7 summarizes the most important SGS parameters of the tested anti-graffiti coating systems.
The tested anti-graffiti coating systems had averaged mean arithmetic surface roughness deviations from the average surface area $\mathrm{Sa}=$ $6.6 \div 26.9 \mathrm{~nm}$. Samples of S235 carbon steel sanded with P80 grain sandpaper on which coatings were applied had Sa $=1234.5 \div 1863.2 \mathrm{~nm}$. Parameter $\mathrm{Sa}$ is the basic amplitude parameter for quantifying the state of the surface being analyzed. A similar trend in the measurement of antigraffiti and S235 coating systems was observed for the quadratic surface roughness Sq, which has a strong correlation with the Sa parameter. As a result of coating application, the surface roughness was significantly reduced.

\section{CONCLUSIONS}

- The mechanical properties of each system are comparable and do not depend on the antigraffiti clearcoat used, whereas the BO100AGR is characterized by the highest hardness,

- Corrosion resistance for each system exceeds $1000 \mathrm{~h}$ in a salt chamber test using a 5\% saline solution,

- Tested rolling stock systems has similar fire test results, which means that anti-graffiti

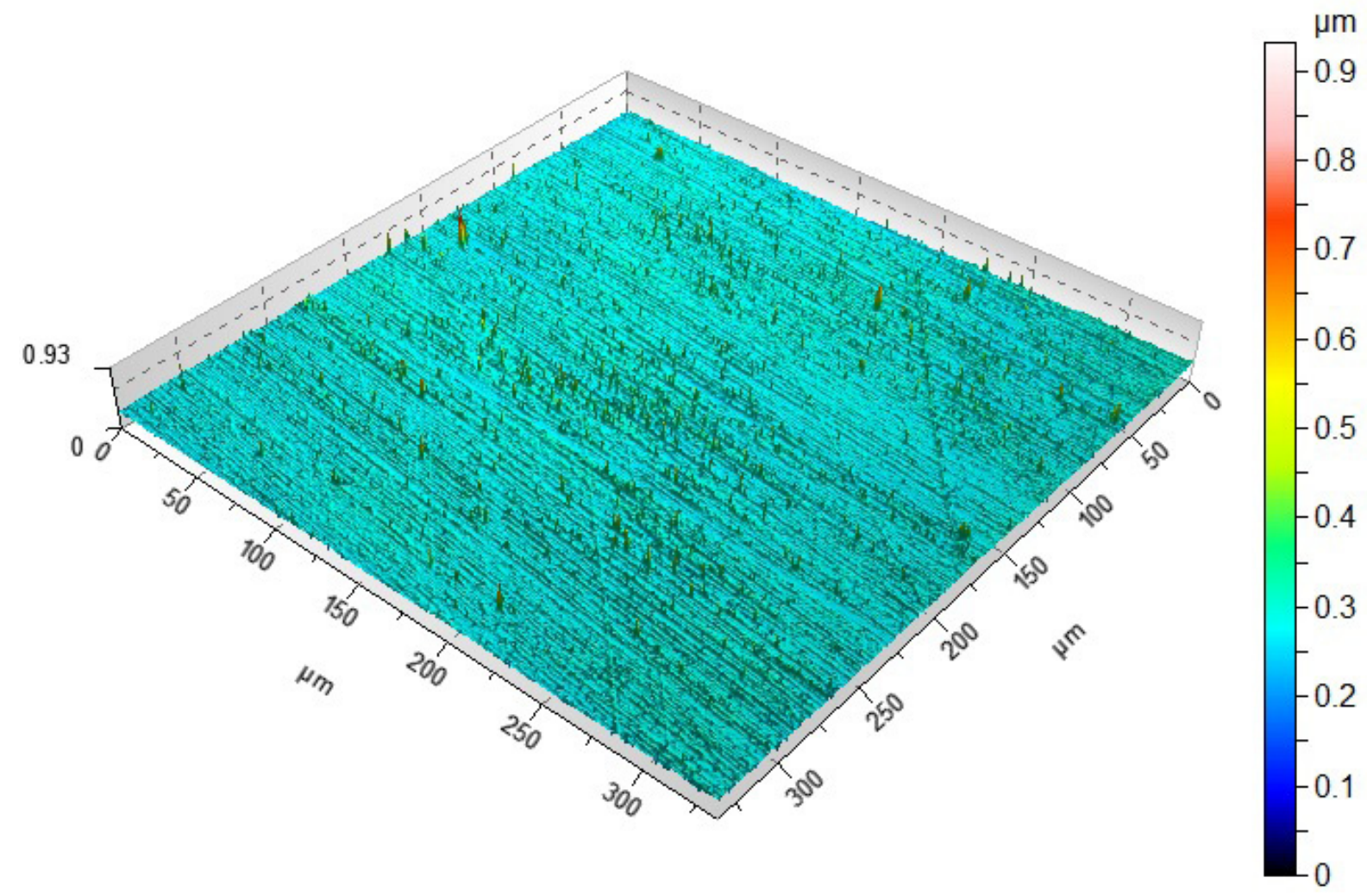

Fig. 4. Isometric view of the surface roughness of the anti-graffiti XPC 60012 coating system 


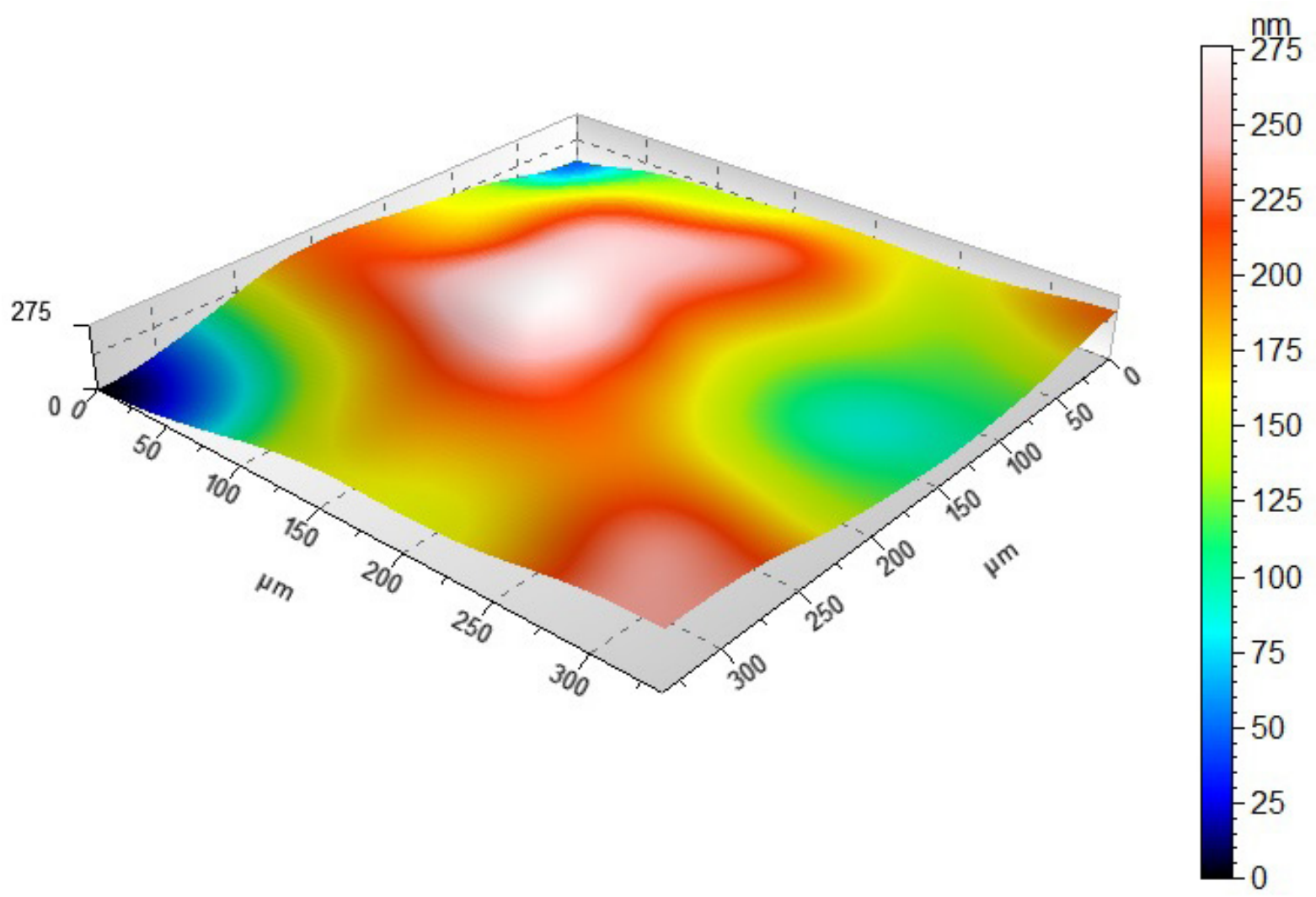

Fig. 5. Isometric view of the waviness surface of the anti-graffiti XPC 60012 coating system

Table 7. Averaged parameters of the surface geometric structure of anti-graffiti coating systems

\begin{tabular}{|c|c|c|c|c|}
\hline \multirow{2}{*}{ SGS } & \multicolumn{4}{|c|}{ System } \\
\cline { 2 - 5 } Parameters & XPC 60011 & XPC 60012 & XPC 60036 & BO100-AGR \\
\hline Sq [nm] & 73.3 & 18.9 & 33.0 & 11.8 \\
\hline Ssk & 0.87 & 3.96 & 3.66 & 155.9 \\
\hline Sku & 20.9 & 64.7 & 52.6 & 501.9 \\
\hline Sp [nm] & 840.4 & 647.5 & 863.9 & 277.8 \\
\hline Sv [nm] & 791.2 & 283.8 & 413.9 & 779.6 \\
\hline Sz [nm] & 1631.6 & 931.3 & 1277.9 & 6.6 \\
\hline Sa [nm] & 26.9 & 11.4 & 16.5 & \\
\hline
\end{tabular}

clearcoat does not affect the deterioration of flammable properties,

- The BO100-AGR is characterized by the best anti-adhesive properties compared to the other three anti-graffiti coating systems. The BO100-AGR anti-adhesie properties can be significant in potential applications on rail vehicles,

- The high values of the slope coefficient of the surface of the Sku (kurtosis) indicate the low dispersion of the ordinate surfaces. The positive values of the asymmetry coefficient of the surface Ssk (skewness) show that making faces with a smooth surface without deep crack.

\section{REFERENCES}

1. Adamczak S., Makieła W.: Analyzing variations in roundness profile parameters during the wavelet decomposition process using the matlab environment. Metrology and Measurement Systems, 2011, XVIII, 1, 25-34.

2. Adamczak S., Miko E., Cus F.: A model of surface roughness constitution in the metal cutting process applying tools with defined stereometry. Strojniski Vestnik-Journal of Mechanical Engineering, 2009, 55, 45-54.

3. EN ISO 5659-2 Plastics - Smoke generation-Part 2: Determination of optical density by a single-chamber.

4. ISO 5658-2:2006 Reaction to fire tests - Spread 
of flame - Part 2: Lateral spread on building and transport.

5. ISO 5660-1 Reaction-to-fire tests - Heat release, smoke production and mass loss rate - Part 1: Heat release rate (cone calorimeter method).

6. Kłonica M., Kuczmaszewski J.: Determining the value of surface free energy on the basis of the contact angle. Advances in Science and Technology Research Journal, 2017, 11, 1, 66-74.

7. Miller T., Adamczak S., Świderski J., Wieczorowski M., Łętocha A., Gapiński B.: Influence of temperature gradient on surface texture measurements with the use of profilometry. Bulletin of the Polish Academy of Sciences, 2017, 65, 1, 53-61.

8. Pietraszek J., Radek N., Bartkowiak K.: Advanced statistical refinement of surface layer's discretization in the case of electro-spark deposited carbideceramic coatings modified by a laser beam. Solid State Phenomena, 2013, 197, 198-202.

9. Pietraszek J.: Fuzzy regression compared to classical experimental design in the case of flywheel assembly. Lect. Notes Artif. Int., 2012, 726, 310-317.

10.PN EN 45545-2 Kolejnictwo. Ochrona przeciwpożarowa w pojazdach szynowych. Część 2: Wymagania dla materiałów i elementów w za- kresie właściwości ogniowych.

11. Radek N., Konstanty J., Scendo M.: The electrospark deposited WC-Cu coatings modified by laser treatment. Archives of Metallurgy and Materials, 2015, 60, 2579-2584.

12. Radziszewska-Wolińska J.: Fire properties of anticorrosion coatings to rolling stock. Czasopismo Techniczne, 2016, 3-M, 79-86.

13. Radziszewska-Wolińska J.: Development of requirements for fire protection of rolling stock in Poland and its comparison with EN 45545. Problemy Kolejnictwa, 2013, 57, 160, 109-119.

14. Rudawska A., Jacniacka E.: Analysis for determining surface free energy uncertainty by the OwenWendt method. International Journal of Adhesion \& Adhesives, 2009, 29, 451-457.

15. Scendo M., Radek N., Trela J.: Influence of laser treatment on the corrosive resistance of $\mathrm{WC}-\mathrm{Cu}$ electrospark coatings. International Journal of Electrochemical Science, 2013, 8, 9264-9277.

16. Zdziennicka A., Szymczyk K., Jańczuk B.: Correlation between surface free energy of quartz and its wettabilit by aqueous solutions of nonionic, anionic and cationic surfactants. Journal of Colloid and Interface Science, 2009, 340, 243-248. 